\title{
Orders Exhibited by Ensemble of Headless Spins Preferring Twisted Alignment: Phase Diagram of Extended Maier-Saupe Model on Simple Cubic Lattice
}

\author{
Kazuya SAito, Mafumi Hishida, and Yasuhisa Yamamura \\ Department of Chemistry, Faculty of Pure and Applied Sciences, \\ University of Tsukuba, Tsukuba, Ibaraki 305-8571, JAPAN
}

\begin{abstract}
The effect of the preference for nonparallel alignment without a preferred twist sense of neighboring headless spins is examined through computer simulations on extended Maier-Saupe models on the simple cubic lattice with/without next-nearest-neighbor interactions. Phase diagrams containing four phases are constructed for a varying degree of the preference for the local twist and for a strength of the next-nearest-neighbor interaction. It is shown that the preference for the local twist brings about not only the instability for the nematic (uniaxial) order but also that for a spatial order to reduce the frustration arising from the local twist. The next-nearest-neighbor interaction can produce even a weak spatial order of local chirality. The results are discussed in relation to the formation of a liquid crystalline gyroid phase and chiral phases in ensembles consisting of antispindle molecules that are axially symmetric and achiral.
\end{abstract}

\section{Introduction}

Spin systems have served as representative models for studying phase transitions and critical phenomena. By referring to the exchange interaction between (quantum) spins on neighboring atoms, usually, interaction between spins, $i$ and $j$, are expressed as $-J_{i j} \boldsymbol{s}_{i} \cdot \boldsymbol{s}_{j}$, and the spontaneous magnetization $\langle\boldsymbol{s}\rangle$, where $\langle\cdot\rangle$ indicates the average over the system, is used as an order parameter to characterize the order (for a ferroic interaction, $J_{i j}>0$ ). However, if a spin is headless, i.e., has no distinction between its head and tail, the spontaneous magnetization has no meaning. In this case, the so-called nematic order parameter $\left\langle P_{2}(\cos \theta)\right\rangle$ becomes important, where $P_{2}(\cdot)$ and $\theta$ are the second-order Legendre polynomial and an angle between a spin orientation and the uniaxial axis, respectively. By virtue of its functional form (see Appendix), a simple meanfield theory can be constructed if the following interaction is assumed for neighboring spins,

$$
V(\theta)=-V_{0} P_{2}\left(\cos \theta_{i j}\right)
$$

with $V_{0}>0$ and $\theta_{i j}$ is the angle between two spins, $i$ and $j$. This mean-field theory is known as the Maier-Saupe theory $^{1)}$ of nematic liquid crystals, which is the most simple liquid crystal with only the orientational order of molecules. As a spin model, the Maier-Saupe model on lattices is a kind of Heisenberg model of headless spins. The model on a simple cubic lattice exhibits a first-order transition between the disordered (isotropic) and the uniaxially ordered (nematic) phases, ${ }^{2,3)}$ in accordance with Landau's thermodynamic phenomenology of phase transitions. $\left.{ }^{4}\right)$

In this study, the effect of preference for the twisted alignment between neighboring spins is investigated by extending the Maier-Saupe model. ${ }^{1}$ Various extensions of the model have already been attempted in the field of liquid crystals such as the inclusion of a higher even-order Legendre polynomial in the intermolecular potential to see its possible effects (while keeping the parallel alignment most stable), ${ }^{5}$ or the inclusion of an odd-order term [e.g., $P_{1}(\cos \theta)$ ] to describe the formation of a cholesteric (chiral nematic) phase consisting of chiral molecules. ${ }^{6}$ ) Differently from these, the extension in this study is made so as to express the twist without a preferred twist sense (handedness), considering recent experimental reports of chiral phases formed by seemingly achiral molecules. ${ }^{7-10)}$ Although it may be difficult to rationalize such extension for traditional spin systems, the extension gives a hint for understanding exotic liquid crystalline phases through an intuitive mapping between the property of the interaction potential and the molecular shape. An extension of the XY-model, which is similar in some sense, has recently been studied ${ }^{11,12)}$ while having main interests on not changing the direction of the preferred orientation but increasing the number of preferred orientations. It is, however, difficult to discuss in comparison with real liquid crystals because of not only the reduced degree of freedom but also the presence of the distinction between the head and the tail of a treated spin, although qualitatively similar phase diagrams were proposed.

In this paper, a phase diagram of a model capable of expressing preferences to a twisted alignment of neighboring headless spins is reported for a lattice model with nearestneighbor interaction. An effect of next-nearest-neighbor interaction is also studied. It is attempted to deduce insights on real liquid crystalline systems from the simulation results. This paper is organized as follows: in Sec. 2, we describe the model and simulation procedures. The results of simulations, phase transitions, and orders established are described in detail separately for without (Sec. 3.1), and with (Sec. 3.2) positive or negative next-nearest-neighbor interactions. Simulation results are summarized in Sec. 4.1. A discussion is given on real liquid crystalline systems in Sec. 4.2, and Conclusion follows.

\section{Model and Simulation Procedure}

Headless spins are assumed to sit on lattice sites of a simple cubic lattice with a lattice spacing of unity. The preference for the twisted alignment without a preferred twist sense for neighboring spins is expressed by the following interaction between the nearest neighbors $(0 \leq r \leq 1)$ :

$$
v(\theta)=\frac{V(\theta)}{V_{0}}=-\left[(1-r) P_{2}(\cos \theta)-r \frac{7}{3} P_{4}(\cos \theta)\right] .
$$




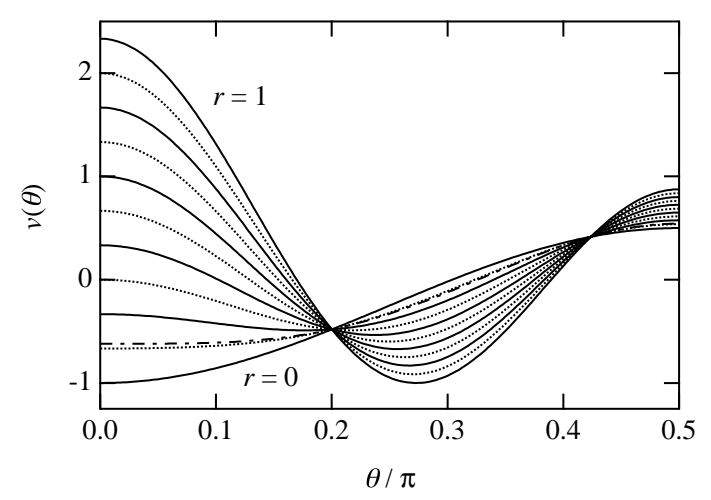

Fig. 1. Potential function $v(\theta)\left[=V(\theta) / V_{0}\right]$ as a function of $r$ (solid and dotted curves alternately by a step of $\Delta r=0.1)$. The dot-dashed curve represents $v(\theta)$ with $r=r_{\mathrm{c}}\left(=\frac{9}{79}\right)$.

Here, $P_{4}(\cdot)$ is the 4 th-order Legendre polynomial. The factor $\frac{7}{3}$ in the second term is a normalization for the perfect order driven solely by it ( $r=1$, see Appendix). The change in the shape of interaction potential [Eq. (2)] is shown in Fig. 1. The $\theta_{\min }$ that minimizes $v(\theta)$ grows from 0 in $r \leq r_{\mathrm{c}}=\frac{9}{79}$ to $\arctan (2 / \sqrt{3})<\frac{\pi}{2}$ at $r=1$. A finite $\theta_{\min }$ means that the interaction is not ferroic. Since two senses of twist (left and right) are energetically equivalent, some alternate order may be expected on the simple cubic lattice. The hump of potential at $\theta=0$, which may serve as a measure of a temperature of phase transition(s) owing to its emergence, grows as a function of $r$ for $r>r_{\mathrm{c}}$, as shown in Fig. 2.

The effect of the next-nearest-neighbor interaction is also examined for limited cases assuming

$$
\frac{V_{\mathrm{NNN}}(\theta)}{V_{0}}=-v_{\mathrm{NNN}} P_{2}(\cos \theta)
$$

Monte Carlo simulations were performed using the Metropolis algorithm. ${ }^{13)}$ The size of the ensembles was $L^{3}$ with $L=30,40,50$, or 60 . The smallest size was mainly used while confirming its consistency with fewer results of larger ensembles including temperature variation. Models taking the next-nearest-neighbor interaction into account required a larger $L$. Indeed, the comparison among the results up to $L=$ 60 was necessary to obtain the reliable result for $v_{\mathrm{NNN}}=0.05$. The cyclic boundary condition was imposed on ensembles. A trial orientation of a molecule without the distinction of the head and tail is uniformly generated at each step. At each temperature, 20000 steps, each of which consists of $L^{3}$ trials, were used to calculate equilibrium (averaged) quantities after 50000 equilibration steps. The attainment of equilibrium was confirmed through coincidences among equilibrium quantities starting from various initial configurations. Note that detailed analyses on transition behaviors such as the precise location of the transition and critical behaviors are not made in this study because these are beyond the present scope. An idea on the temperature steps used in simulations can be obtained from the result with the next-nearest-neighbor interaction (Fig. 7). In this paper, the temperature is measured in terms of energy, i.e., $k_{\mathrm{B}}=1$, and all extensive quantities such as the averaged energy $\langle E\rangle$ and the heat capacity $C$ are given for a single spin. Although $C$ can be calculated both from the fluctuation of energy, $C=\left(\left\langle E^{2}\right\rangle-\langle E\rangle^{2}\right) T^{-2}$, and as a tem-

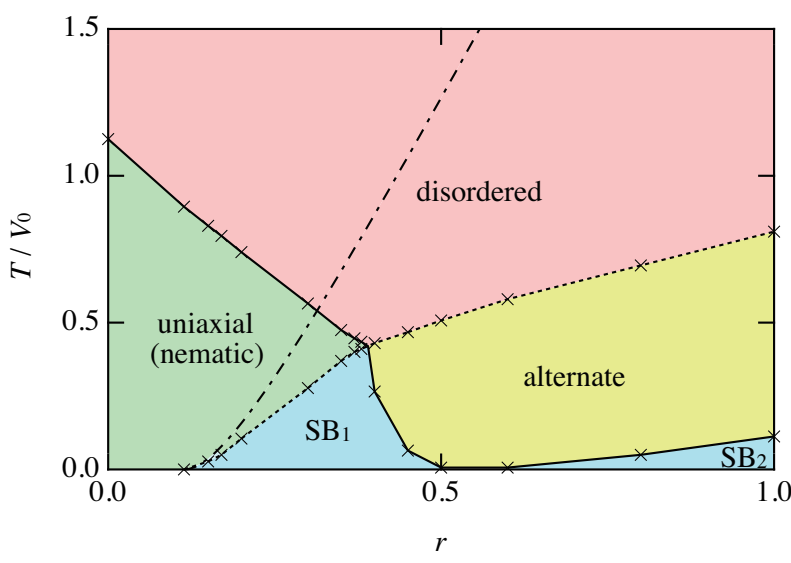

Fig. 2. (Color online) Phase diagram of extended Maier-Saupe model on simple cubic lattice as a function of $r\left(v_{\mathrm{NNN}}=0\right)$. SB phases possess both uniaxial and alternate orders. Solid line, first-order phase transition driven by nematic instability; dotted line, second-order phase transition with alternate twist order. The dot-dashed line indicates the height of the energy hump at $\theta_{\mathrm{NN}}=0$ in the nearest-neighbor interaction [Eq. (2)].

perature derivative of the energy, $C=d\langle E\rangle / d T$, those via the latter are depicted in this paper because of their smoothness, which is preferable for detecting tiny anomalies.

Since the system considered is symmetric concerning the uniform rotation of spins, the simulation results will be given after spins are uniformly rotated so as to align the principal axis of the largest component of the tensor nematic order parameter, i.e., the so-called "director" of the nematic order, along the $z$-axis. Hereafter, the $\theta$ without subscripts is used to denote the angle between the spin and the nematic director (z-axis). The nematic order parameter is represented as $\left\langle P_{2}(\cos \theta)\right\rangle$, accordingly. On the other hand, the subscript "NN" represents nearest neighbors. The average of angles between nearest neighbors is written as $\left\langle P_{2}\left(\cos \theta_{\mathrm{NN}}\right)\right\rangle$.

To obtain the information on possible spatial orders, a "diatomic molecule" consisting of two point scatters (bond length being 0.2 in the unit of the lattice spacing) was put at each lattice point according to the simulated spin configuration. A "structure factor", which is a Fourier component inherent in a configuration, was then calculated. All structure factors reported in this paper were calculated on the basis of the respective snapshot and not averaged over snapshots.

\section{Results}

\subsection{Case without next-nearest-neighbor interaction}

Simulations of models without the next-nearest-neighbor interaction $\left(v_{\mathrm{NNN}}=0\right)$ were comprehensively performed over the entire range of $r$. The $r$ 's simulations performed can be read from the phase diagram (Fig. 2), which seemingly contains five phases. The selected results of temperature dependences of the nematic order parameters are shown in Fig. 3. The phase diagram is divided into four regions depending on $r$. These are described separately.

\subsubsection{Region I: $r \leq r_{\mathrm{c}}$}

In this region, the perfect nematic order $\left(\theta_{\min }=0\right)$ is realized at $T=0$. The lattice Maier-Saupe model was already studied in the literature. $^{2,3)}$ The present result with $r=0$ completely coincides with the reported results: The disordered 


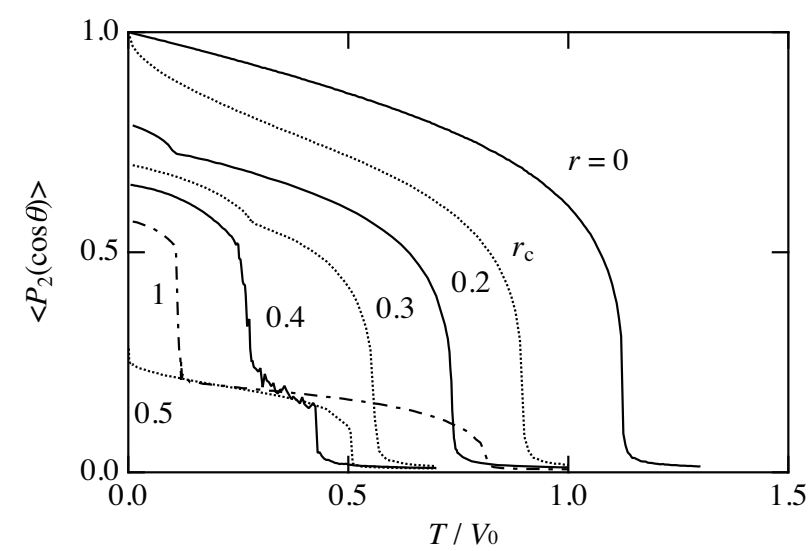

Fig. 3. Temperature dependences of nematic order parameter $\left\langle P_{2}(\cos \theta)\right\rangle$ for selected values of $r\left(v_{\mathrm{NNN}}=0\right)$. Dependences are drawn by curves (solid, dotted or dot-dashed) for clarity although simulations were done rather sparsely in reality.

phase (corresponding to the isotropic liquid) changes to the uniaxialy ordered (nematic) phase at $T_{\mathrm{du}} \approx 1.12 V_{0}$ on cooling. The phase transition is of first order as theoretically revealed on the basis of the asymmetry between positive and negative nematic order parameters. ${ }^{4}$ ) The order of this disordereduniaxial transition remains the same even in $r \geq r_{\mathrm{c}}$. In practice, however, it was difficult to see the symptoms of hysteresis arising from the first-order nature of the transition.

With increasing $r$ from 0 , the interaction potential becomes shallower. The transition temperature monotonically decreases (Fig. 2). The growth of the nematic order on lowering temperature becomes weaker as seen for $r=r_{\mathrm{c}}$ in Fig. 3.

The heat capacity $C$ at low temperatures reflects the excitation spectrum. Note that only the potential energy is taken into calculation in the present treatment. A single classical harmonic oscillator contributes not by 1 including the kinetic energy contribution of $\frac{1}{2}$ but only by $\frac{1}{2}$, accordingly. Since a spin has two degrees of freedom, the limiting value of the heat capacity (at $T=0$ ) is the unity in this region ${ }^{2)}$ except for the close vicinity of $r_{\mathrm{c}}$, where the effective force constants of the restoring force on spins (the second derivative of the interaction by the angle) vanishes. At $r=r_{\mathrm{c}}$, the numerical derivative yields $C=\frac{1}{2}$, which is the magnitude expected for two classical oscillators each with a quartic (not quadratic) potential function.

\subsubsection{Region II: $r_{\mathrm{c}}<r \lesssim 0.38$}

Once $r$ exceeds $r_{\mathrm{c}}$, an energy hump emerges at $\theta_{\mathrm{NN}}=0$ in the interaction (Eq. 2). The distribution of the angle between neighboring spins, $\theta_{\mathrm{NN}}$, changes qualitatively from those with the maximum at $\theta_{\mathrm{NN}}=0$ for $r \leq r_{\mathrm{c}}$. Indeed, the ensemble average $\left\langle\cos ^{2} \theta_{\mathrm{NN}}\right\rangle$ always has a similar magnitude to $\cos ^{2} \theta_{\text {min }}$ with small anomalies at phase transitions. Such a change, however, does not necessarily accompany any phase transitions.

Because of the hump at $\theta_{\mathrm{NN}}=0$, the perfect nematic order cannot be the ground state of the system for $r>r_{\mathrm{c}}$. A phase transition occurs at a lower temperature as seen as a kink in the temperature dependence of the nematic order parameter in Fig. 3 for $r=0.2$ (at $T_{\mathrm{uSB}} \approx 0.10 V_{0}$ ) and $r=0.3$ (at
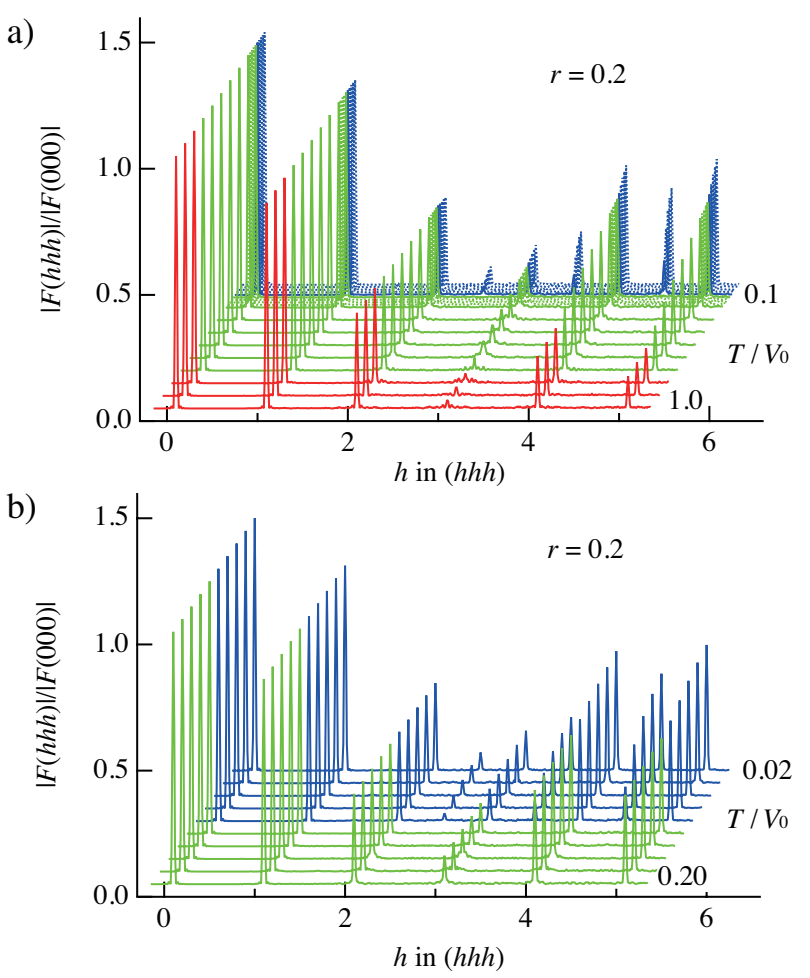

Fig. 4. (Color online) Temperature variation of structure factor along $(h h h)$ at $r=0.2\left(v_{\mathrm{NNN}}=0\right)$. (a) whole temperature region $\left(\Delta T=0.1 V_{0}\right)$, (b) details at low temperatures $\left(\Delta T=0.02 V_{0}\right)$. On cooling below a lower transition $\left(T_{\mathrm{uSB}} \approx 0.105 V_{0}\right)$, superlattice components $\left(h=k+\frac{1}{2}, k=0,1,2 \ldots\right)$ grow in the $\mathrm{SB}_{1}$ phase.

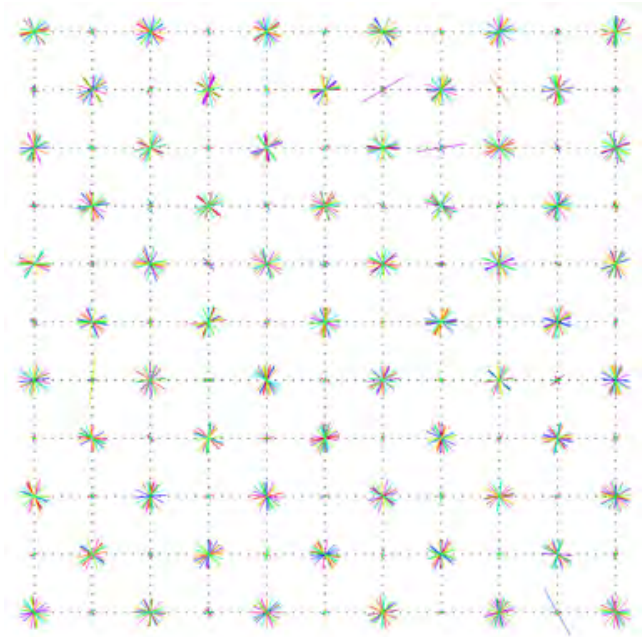

Fig. 5. (Color online) Projection of 15 even-numbered layers in snapshots on the $x y$-plane in an $11 \times 11$-region for $r=0.2, v_{\mathrm{NNN}}=0$ at $T=0.02 V_{0}$ (in $\mathrm{SB}_{1}$ phase ). Different colors of segments (molecules) distinguish layers they belong to.

$\left.T_{\mathrm{uSB}} \approx 0.28 V_{0}\right)$.

To obtain some information of the change in the order, structure factors were examined. The disordered and uniaxial phases give only Bragg peaks arising from the lattice properties of the model. Upon the lower transition, superlattice components appear only in $\{h h h\}$ directions shown in Fig. 4. Their locations, $\{h h h\}$ with $h=k+\frac{1}{2}, k=0,1,2 \ldots$, indicate that some structural order corresponding to the "rock 


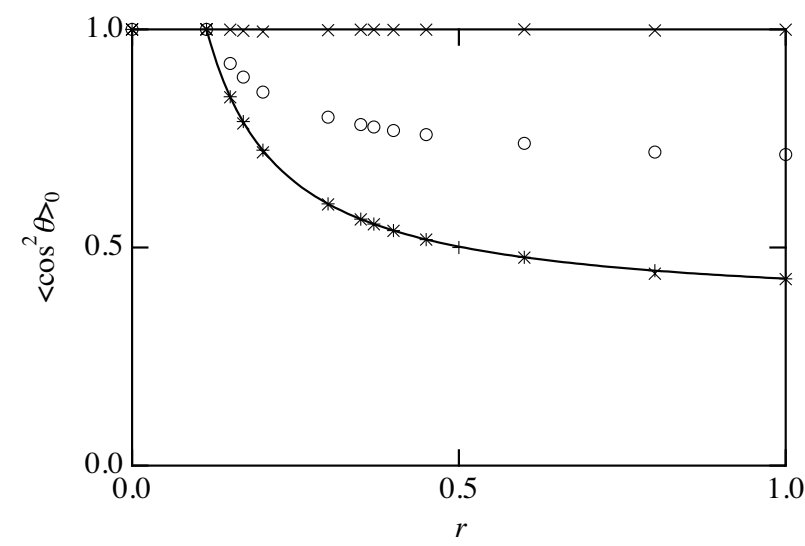

Fig. 6. Low-temperature (limiting) values of averages of various $\cos ^{2} \theta$ values as a function of $r\left(v_{\mathrm{NNN}}=0\right)$. Open circle, $\left\langle\cos ^{2} \theta\right\rangle_{0}=$ $\left(2\left\langle P_{2}(\cos \theta)\right\rangle_{0}+1\right) / 3$; plus sign, $\left\langle\cos ^{2} \theta_{\mathrm{NN}}\right\rangle_{0}$; cross, $\left\langle\cos ^{2} \theta\right\rangle_{0} \pm \sqrt{\left\langle\Delta \cos ^{2} \theta\right\rangle_{0}}$, where $\left\langle\Delta \cos ^{2} \theta\right\rangle_{0}=\left\langle\cos ^{4} \theta\right\rangle_{0}-\left\langle\cos ^{2} \theta\right\rangle_{0}^{2}$. The solid curve represents $\cos ^{2} \theta_{\min }$, where $\theta_{\min }$ gives the minimum of $v(\theta)$.

salt" structure emerges upon the transition. Namely, a spatial order with the alternation exists in the low-temperature phase as in alloys such as brass, a classical example of orderdisorder transition. Hereafter, the phase is specified as the $\mathrm{SB}_{1}$ phase. An example of a snapshot of the simulation ensemble in the $\mathrm{SB}_{1}$ phase is shown in Fig. 5. The existence of the alternate order is clearly visible. In Fig. 6, limiting values of averages of some " $\cos ^{2} \theta$ "s at $T=0$ are plotted as a function of $r$. That of the angles between the uniaxial axis and the molecular figure axis, $\left\langle\cos ^{2} \theta\right\rangle_{0}$, lies on neither $\cos ^{2} \theta_{\min }$ nor $\cos ^{2}\left(\theta_{\min } / 2\right)$, which is expected if two types of sites are related by a mirror symmetry. When the standard deviation of $\cos ^{2} \theta$, i.e., $\sqrt{\left\langle\Delta \cos ^{2} \theta\right\rangle_{0}}$, is added to and subtracted from $\left\langle\cos ^{2} \theta\right\rangle_{0}$, the results, $\left\langle\cos ^{2} \theta\right\rangle_{0} \pm \sqrt{\left\langle\Delta \cos ^{2} \theta\right\rangle_{0}}$, lie on the line of perfect nematic order $\left(\left\langle\cos ^{2} \theta\right\rangle=1\right)$ and that of $\cos ^{2} \theta_{\text {min }}$. This indicates that one half of spins on one type of sites ("ordered" sites) tend to orient themselves perfectly along the uniaxial axis whereas the other half on the other type of sites ("disordered" sites) are inclined by $\theta_{\min }$ from the axis at $T=0$. This state certainly has the minimum energy with macroscopic degeneracy. There is no mechanism to order the orientation of spins on the latter type of sites because the interaction works only between nearest neighbors. That is, there remains the rotational degrees of freedom around the uniaxial axis $(z)$ for spins on the disordered sites. There is a zero-energy mode in this ground state, accordingly. Reflecting the existence of this mode, the heat capacity $C$ in the lowest temperature region is $\frac{3}{4}\left[=\frac{1}{2}\left(1+\frac{1}{2}\right)\right]$ in contrast to 1 in the uniaxial phase for $r<r_{\mathrm{c}}$.

The temperature of the phase transition between the uniaxial and the $\mathrm{SB}_{1}$ phases increases with $r$. It is reasonable to assume that the transition emerges at $r_{\mathrm{c}}$ because of the emergence of the hump at $\theta_{\mathrm{NN}}=0$. Figure 2 indicates that the thermal energy at the transition temperature is of the same order as but lower than the energy hump at $\theta_{\mathrm{NN}}=0$ in the interaction (Eq. 2). Two phase boundaries (one between the disordered and uniaxial phases and the other between the uniaxial and $\mathrm{SB}_{1}$ phases) encounter at around $r \approx 0.38$.

\subsubsection{Region IIIa: $0.38 \lesssim r \lesssim 0.5$}

In this region, two phase transitions still occur on cooling from the disordered phase. However, the upper transition accompanies not only the evolvement of the small nematic order parameter but also the appearance of superlattice components corresponding to the rock salt structure while the lower transition does an appreciable growth in the nematic order parameter as seen in Fig. 3 for $r=0.4$. The connectivity of phase boundaries and the limiting $\left\langle\cos ^{2} \theta\right\rangle_{0}$, etc. suggest that the phase realized at the lowest temperatures is the $\mathrm{SB}_{1}$ phase. The phase between two transitions is qualitatively the same as the $\mathrm{SB}_{1}$ phase with respects to a finite nematic order parameter and a spatial order of rock salt type. However, it is to be distinguished from the $\mathrm{SB}_{1}$ phase because there is a transition boundary to the $\mathrm{SB}_{1}$ phase. It is thus called an alternate phase while paying attention to the presence of the spatial order. With the increase in $r$, starting from the "quadlple" point, the temperature of the upper transition between the disordered and alternate phases gradually increases whereas that of the lower transition between the alternate and $\mathrm{SB}_{1}$ phases rapidly decreases. The lower boundary reaches nearly the $T=0$ line at slightly larger than $0.5 \mathrm{in} r$. Indeed, the transition temperature at $r=0.5$ is located at around $T_{\mathrm{aSB}} \approx 0.001 V_{0}$, which is the lowest temperature studied. The temperature dependences of quantities such as $\left\langle\cos ^{2} \theta\right\rangle$ cannot be extrapolated to reasonable values expected from results of $r \neq 0.5\left(r \geq r_{\mathrm{c}}\right)$ as shown in Fig. 6. It is unclear at present whether the $\mathrm{SB}_{1}$ phase really vanishes around $r \approx 0.5$.

\subsubsection{Region IIIb: $0.5 \lesssim r$}

In this region, two phase transitions occur on cooling from the disordered phase similarly to Region IIIa. The transition at a higher temperature is certainly that from the disordered phase to the alternate phase. As for the lowest temperature phase, considering the distinct jump in the nematic order parameter and limiting values such as $\left\langle\cos ^{2} \theta\right\rangle_{0}$ and $C=\frac{3}{4}$, it can be said that the phase is quite similar to the $\mathrm{SB}_{1}$ phase in Regions II and IIIb. The phase is called the $\mathrm{SB}_{2}$ phase, accordingly. The temperatures of two phase transitions gradually increase with the increase in $r$.

\subsection{Case with next-nearest-neighbor interaction}

Even if we assume only the steric effect (see Fig. 14), the interaction between not only the nearest but also the nextnearest-neighbors is expected to work in real nonlattice systems. Considering the steric effect, the antiferroic interaction, which prefers the continuous twist in one direction as in the gyroid phase (Fig. 13), is more probable than the ferroic one preferring the zigzag arrangement in one dimension. In this study, however, both types of the next-nearest-neighbor interaction are treated. Simulations were performed only for $r=0.2$ and $v_{\mathrm{NNN}}= \pm 0.05$, which is one order of magnitude smaller than the interaction between nearest neighbors in the sense $z_{\mathrm{NNN}}\left|v_{\mathrm{NNN}}\right| / z_{\mathrm{NN}}=\frac{1}{10}$ with $z_{\mathrm{NN}}=6$ and $z_{\mathrm{NNN}}=12(z$ being the numbers of interacting spins).

In the alternate order (in the alternate, $\mathrm{SB}_{1}$ or $\mathrm{SB}_{2}$ phase), the spins involved in the next-nearest-neighbor interaction sit on equivalent sites. For example, they are on either the ordered or disordered sites in SB phases. Considering the smallness of $\left|v_{\mathrm{NNN}}\right|$, here, we focus on its effect in the $\mathrm{SB}_{1}$ phase 


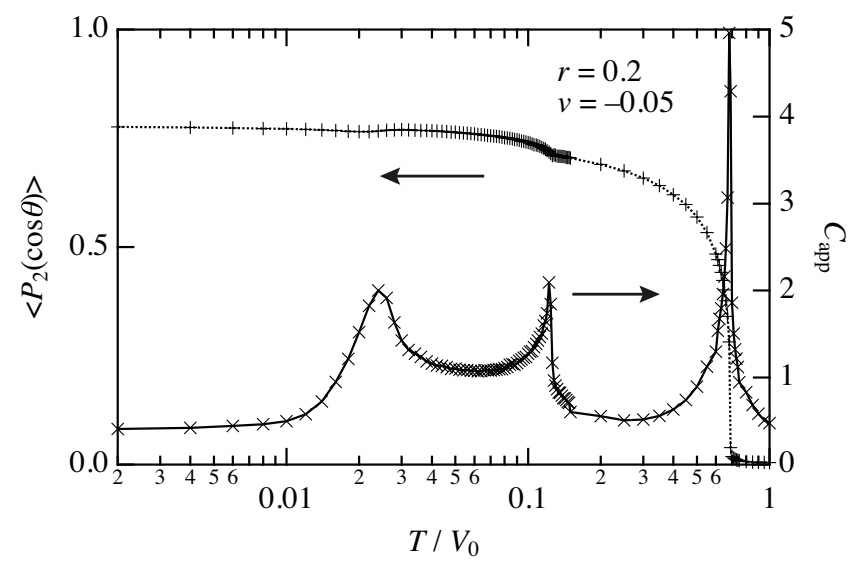

Fig. 7. Temperature dependence of nematic order parameter $\left\langle P_{2}(\cos \theta)\right\rangle$ and apparent heat capacity $C_{\text {app }}=d\langle E\rangle / d T$ obtained by numerical differentiation of energy for $r=0.2$ and $v_{\mathrm{NNN}}=-0.05$.

at sufficiently low temperatures. Then, $v_{\mathrm{NNN}}$ between spins on the disordered sites works as the interaction to induce the orientational order among them. If the presence of spins on the ordered sites is ignored, the problem can be regarded as a three-dimensional XY-model, which is known to exhibit a phase transition. ${ }^{14)}$ Note that the distinction between the head and the tail of spins "revives" in this mapping (because of $\left.0<\theta_{\min }<\frac{\pi}{2}\right)$ despite its absence for the original spins. However, the transitions induced by the next nearest neighbor interaction are not those purely understood within the context of the three-dimensional XY-model because of the presence of molecules on the ordered sites. Moreover, the effects of $v_{\mathrm{NNN}}$ differ depending on its sign as described below.

\subsubsection{Negative next-nearest-neighbor interaction: $v_{\mathrm{NNN}}=-0.05$}

A negative $v_{\mathrm{NNN}}$ is basically different from the positive one because it perturbs the perfect order of molecules on the ordered sites without it. It is difficult to construct intuitively the ground state that is fully ordered in some sense.

The temperature dependence of the nematic order parameter is shown in Fig. 7. The two phase transitions corresponding to those in cases with $r>r_{\mathrm{c}}$ and $v_{\mathrm{NNN}}=0$ can be clearly recognized at $T_{\mathrm{du}} \approx 0.685 V_{0}$ and $T_{\mathrm{uSB}} \approx 0.125 V_{0}$. Note that the former is decreased while the latter is increased. These are based on the fact that the negative $v_{\mathrm{NNN}}$ destroys the nematic order. Besides, there is a tiny anomaly around $T \approx 0.024 V_{0}$. The apparent heat capacities obtained by the numerical differentiation of the average energy (Fig. 7) exhibit a pronounced anomaly there. It is thus concluded that an additional phase transition is induced by the next-nearest-neighbor interaction.

To obtain insights about the order appearing at the phase transition at the lowest temperature, putting the importance in a chiral order in the three-dimensional XY-model in mind, ${ }^{14)}$ the distribution of the following local chirality is examined:

$$
\chi_{\text {local }, i}=\frac{1}{4} \sum_{j \in \mathrm{NN}^{\prime}}\left[\left(\boldsymbol{r}_{j}-\boldsymbol{r}_{i}\right) \times \omega_{j}\right]_{z},
$$

where the sum runs over four nearest neighbors with the common $z$-coordinate. $\boldsymbol{r}_{j}$ and $\omega_{j}=\left(x_{j}, y_{j}, z_{j}\right)\left(z_{j} \geq 0\right)$ are respectively the position and unit vector expressing the orientation

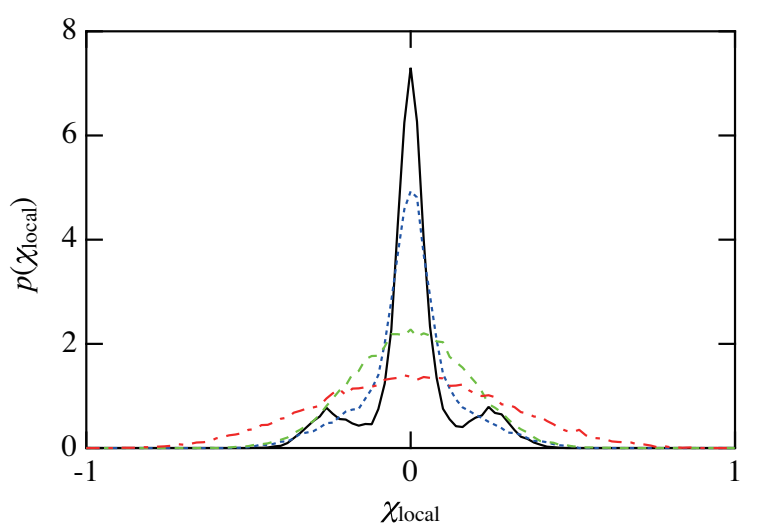

Fig. 8. (Color online) Distribution of local chirality $\chi_{\text {local }}$ estimated from respective snapshots for $r=0.2$ and $v_{\mathrm{NNN}}=-0.05$ in disordered phase $(T=$ $V_{0}$, dot-dashed curve), uniaxial phase ( $T=0.5 V_{0}$, dotted curve), $\mathrm{SB}_{1}$ phase ( $T=0.05 V_{0}$, broken curve), and chiral-segregation phase $\left(T=0.005 V_{0}\right.$, solid curve).

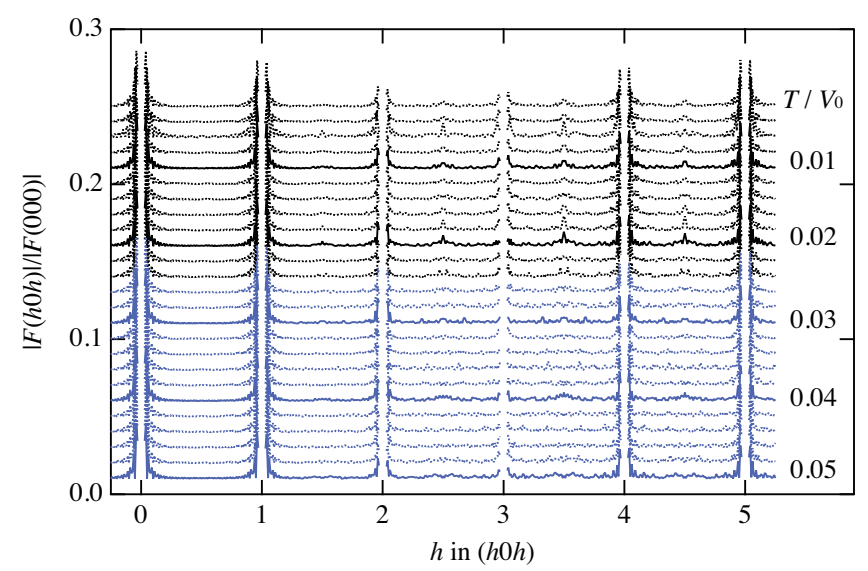

Fig. 9. (Color online) Temperature variation of structure factor along $(h 0 h)$ at $r=0.2$ and $v_{\mathrm{NNN}}=-0.05$. Strong Bragg peaks are omitted for clarity.

of the $j$-th spin, and $[\cdot]_{z}$ the $z$ component (along the uniaxial axis of the nematic order) of the vector [·] . The distribution functions of $\chi_{\text {local }}$ are shown in Fig. 8 at selected temperatures corresponding to different phases. Although the distribution function is symmetric in all phases, distinct peaks (around $\chi_{\text {local }} \approx 0.24$ ) appear in addition to the central peak in the lowest temperature phase. In this respect, the growth of the local chirality is characteristic of the phase. This form of distribution can be rationalized by considering the existence of two types of sites (ordered and disordered sites in the $\mathrm{SB}_{1}$ phase). Namely, the "disordered" sites predominantly contribute to the central peak whereas the ordered sites contribute to the two outer peaks owing to the twist of molecules on the neighboring "disordered" sites.

The local chirality forms a weak spatial order as evidenced by the appearance of additional superlattice components at $\{h 0 h\}$ with $h=k+\frac{1}{2}(k=0,1,2, \ldots)$ (Fig. 9), which is different from those observed in the alternate and SB phases. The system is now biaxial. However, these data are still insufficient to have an idea concerning the spatial order of the lowest temperature phase. Indeed, the distribution of the local chirality exhibits appreciable broadness even at the lowest temperature 


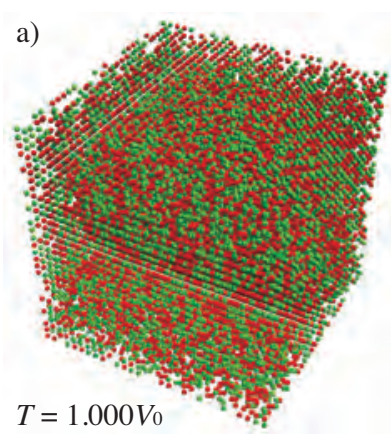

b)

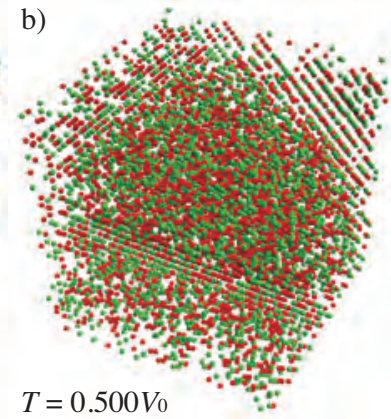

c)

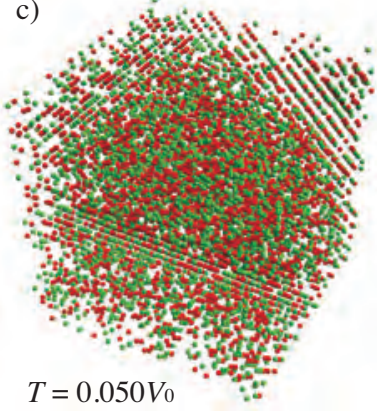

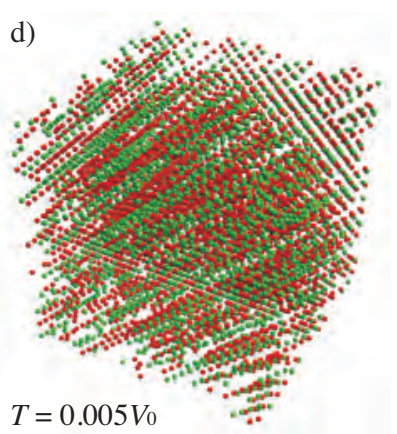

Fig. 10. (Color online) Spatial distribution of local chirality $\chi_{\text {local }}$ according to respective snapshots for $r=0.2$ and $v_{\mathrm{NNN}}=-0.05$ in disordered phase $\left(T=V_{0}\right)$, uniaxial phase $\left(T=0.5 V_{0}\right), \mathrm{SB}_{1}$ phase $\left(T=0.05 V_{0}\right)$, and chiral-segregation phase $\left(T=0.005 V_{0}\right)$. The red and green colors are different in the sign of $\chi_{\text {local }}$ with $\left|\chi_{\text {local }}\right|>0.15$.

studied $\left(T=0.002 V_{0}\right)$. Note that the distribution should consist of "spikes" if the spatial order is perfect (perfect crystal). Thus, an idea is looked for snapshots. The snapshots of simulations are shown as spatial distributions of the local chirality in Fig. 10, where the threshold is set as $\left|\chi_{\text {local }}\right|>0.15$ considering the dip in the distribution in the lowest temperature phase. Note that a slight shift in the threshold does not alter the impression of the spatial distribution. As clearly seen, the distributions of respective handedness (colors) are random in disordered, uniaxial, and $\mathrm{SB}_{1}$ phases, whereas linear and parallel alignments are recognized in the lowest temperature phase. The lowest temperature phase with negative $v_{\mathrm{NNN}}$ is thus characterized as a chiral-segregation phase.

Heat capacities at low temperatures are estimated as ca. 0.5 between $T=0.002 V_{0}$ and $0.01 V_{0}$, which is half of that of the normal "ordered" state and suggests some special situation in the chiral-segregation phase. However, neither the qualitative description of the order nor mechanisms for its emergence can be suggested at present.

\subsubsection{Positive next-nearest-neighbor interaction: $v_{\mathrm{NNN}}=0.05$}

A positive $v_{\mathrm{NNN}}$ aligns next-nearest-neighbors. The perfect order of spins on the ordered sites in the $\mathrm{SB}_{1}$ phase is compat-

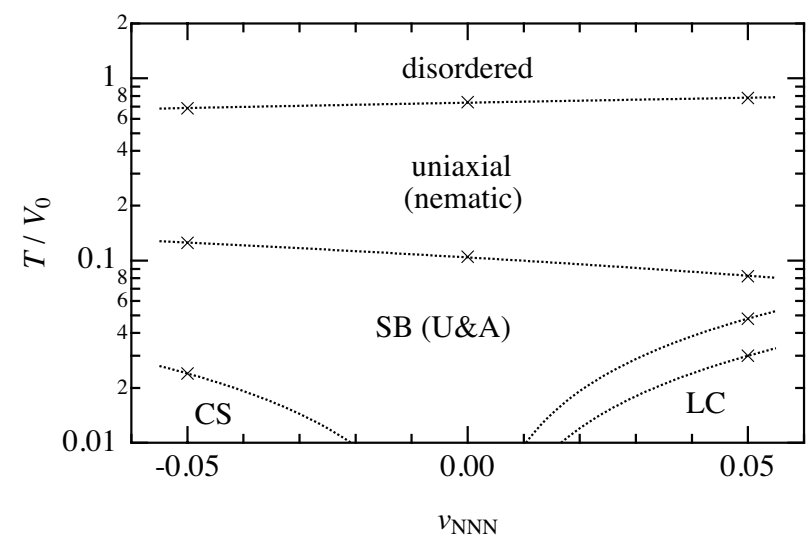

Fig. 11. Phase diagram of extended Maier-Saupe model on cubic lattice as a function of $v_{\mathrm{NNN}}(r=0.2)$. The SB phase possesses both uniaxial (U) and alternate (A) orders. CS and LC stand for "chiral-segregation" and "locally chiral," respectively. Two upper phase boundaries are linearly drawn while those at lowest temperatures are drawn assuming $T_{\mathrm{trs}} \propto\left|v_{\mathrm{NNN}}\right|$. ible with it. Thus, we can naïvely imagine the configuration in the ground state with the minimum energy: perfect alignments of spins on two types of sites with the $\theta_{\min }$ as the angle between them. If, in this limiting situation (at $T=0$ ), the bisector of the angle is aligned along one of the unit vectors of the cubic lattice, two sites become equivalent in the sense that they are related by some symmetry operation. However, the result of the simulation is quite complicated and difficult to rationalize intuitively.

Simulations for $v_{\mathrm{NNN}}=0.05$ indicated the occurrences of two similar phase transitions to those in the case of $v_{\mathrm{NNN}}=$ -0.05 . Their temperatures are $T_{\mathrm{du}} \approx 0.78 V_{0}$ for the transition between disordered and uniaxial phases, and $T_{\mathrm{uSB}} \approx 0.082 V_{0}$ for that between the uniaxial and $\mathrm{SB}_{1}$ phases. The shifts of these transitions from the case without the next-nearestneighbor interaction are almost the same in their absolute magnitude but opposite in sign in comparison with the case of $v_{\mathrm{NNN}}=-0.05$. This supports the reasoning for the shifts given for $v_{\mathrm{NNN}}=-0.05$. Thus, we can write the dependences as $d T_{\mathrm{du}} / d v_{\mathrm{NNN}} \approx 0.95$ and $d T_{\mathrm{uSB}} / d v_{\mathrm{NNN}} \approx-0.43$. The phase diagram against $v_{\mathrm{NNN}}$ at $r=0.2$ is shown in Fig. 11 .

In the low-temperature region, the behavior is rather mysterious. The temperature dependences of the nematic order parameter and apparent heat capacity are shown in Fig. 12. Two characteristic temperatures are clearly identified in the heat

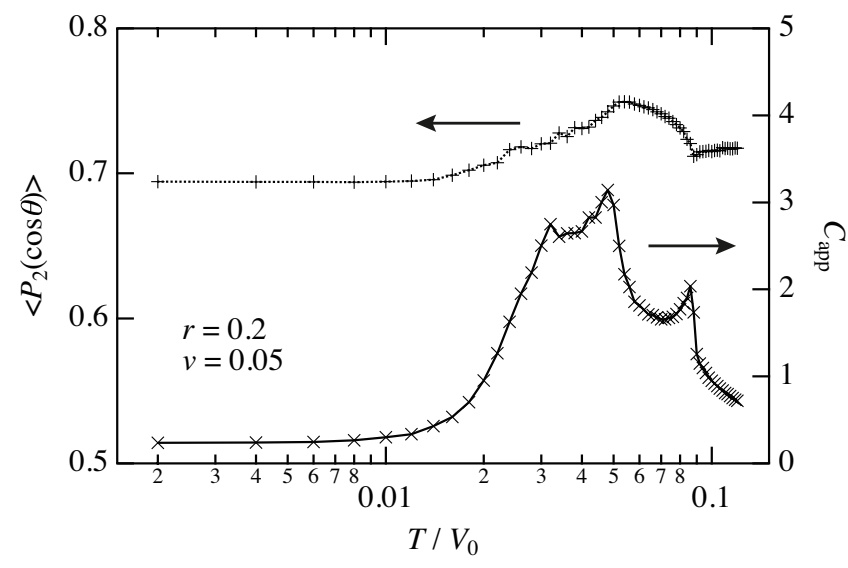

Fig. 12. Temperature dependence of nematic order parameter $\left\langle P_{2}(\cos \theta)\right\rangle$ and apparent heat capacity $C_{\text {app }}=d\langle E\rangle / d T$ obtained by numerical differentiation of energy for $r=0.2$ and $v_{\mathrm{NNN}}=0.05$. 
capacity at $T \approx 0.048 V_{0}$ and $0.030 V_{0}$. Alhough it suggests the existence of two phase transitions, no symptom of a new spatial order was identified in structure factors although the local chirality [Eq. (4)] below the higher transition temperature $\left(T \approx 0.048 V_{0}\right)$ exhibits similar distributions to the case of $v_{\mathrm{NNN}}=-0.05$ shown in Fig. 8. Furthermore, inspections over snapshots at very low temperatures (down to $T=0.001 V_{0}$ ) indicate that the degree of the orientational order of the spins on the ordered sites is not so high, or rather lower than that shown in Fig. 5. The detailed characterization of the orders at low temperatures is left for a future work.

Heat capacities at low temperatures are estimated as ca. 0.25 between $T=0.002 V_{0}$ and $0.01 V_{0}$, which is only a quarter of that of an "ordered" state with two degrees of freedom (described by a harmonic oscillator for each) per spin. The difference in the low-temperature heat capacity between $v_{\mathrm{NNN}}= \pm 0.05$ clearly indicates that the lowest temperature phases are significantly different from each other. Concerning the small heat capacity, two possibilities can be suggested. One is that there is zero-energy mode(s) as in the case of the $\mathrm{SB}_{1}$ phase without the next-nearest-neighbor interaction, while the other is that the system is in a glassy state below some temperature. The fact that larger systems (up to $L=60$ ) were necessary to assure the consistency of the simulation results might be a symptom of the latter possibility.

\section{Discussion}

\subsection{Summary and implication of simulation results}

In this study, Monte Carlo simulations were performed on extended Maier-Saupe models [Eqs. (2) and (3)] on a simple cubic lattice in order to see the effects of the local preference for the nonparallel (twisted) alignment of neighboring headless spins while retaining their axial symmetry. The simulations revealed the presence of two types of instabilities (tendency to a respective order) depending on the degree of the preference if only the nearest neighbor interaction is taken into account. The weak next-nearest-neighbor interaction induces other instabilities with different spatial orders depending on its sign.

The tendency to the uniaxial (nematic) order brings about a first-order phase transition while a continuous transition results from that to the alternate order, which appears to resolve the frustration in the local twists. Two instabilities behave almost independently. The phase sequence on cooling is thus either disordered $\rightarrow$ uniaxial $\rightarrow$ uniaxial and alternate $(\mathrm{SB})$ or disordered $\rightarrow$ alternate $\rightarrow$ SB. It may be sufficiently interesting by itself that a simple interaction between nearest neighbors can bring about two successive phase transitions upon temperature variation.

The nature of the transition between the disordered and uniaxial (nematic) phases is protected by the symmetry property of the primary order parameter, ${ }^{4)}$ i.e., the nematic order parameter. As long as the perfect order is preferred in the interaction $\left(r \leq r_{\mathrm{c}}\right.$ and $\left.v_{\mathrm{NNN}}=0\right)$, the magnitude of the order parameter in the ground state is trivially unity while its temperature dependence is not universal but dependent on the shape of the interaction. Even those convex downward are possible.

Once a hump at the parallel alignment emerges in the interaction, the local arrangement between neighbors becomes a twisted one at a sufficiently low temperature (depending on the potential). A crossover between the locally twisted and parallel alignments occurs upon temperature variation, but it does not necessarily accompany a phase transition.

The ground state with the uniaxial and alternate order (SB phase) without the next-nearest-neighbor interaction is of the rock salt structure consisting of two kinds of sites (ordered and disordered). At $T=0$, spins on the ordered sites exhibit a perfect uniaxial order while those on the disordered sites are uniformly distributed around the uniaxial axis defined by the former with the tilt angle determined by the interaction preferring the local twist. This order has the macroscopic degeneracy. It is noteworthy that this ground state configuration is possible as long as the lattice structure is bipartite, i.e., contains only even-membered loops of interaction paths. Thus, this type of alternate arrangement is expected to be always the ground state irrespective of lattice structures fulfilling the bipartite property. Studies on non-bipartite lattices such as a triangular lattice in the two-dimension are interesting and will be explored in the future.

The weak next-nearest-neighbor interaction affects almost linearly the temperatures of the upper two phase transitions. The effect can be rationalized by considering its effect on the stability of the uniaxial (nematic) phase. The next-nearestneighbor interaction, irrespective of its sign, induces another phase transition from the SB phase to a phase showing a local chiral order retaining the macroscopic achirality. It implies that the presence of only a nearest neighbor interaction is insufficient to induce a chiral order even if locally. The local chiral order induced by the next-nearest-neighbor interaction forms a weak spatial order if the next-nearest-neighbor interaction does not favor the nematic order $\left(v_{\mathrm{NNN}}<0\right)$. The resultant spatial periodicity seems commensurate with the underlying periodicity of the lattice. Considering helical orders in magnetism where the relative magnitude of interactions between nearest and next-nearest-neighbors governs the periodicity of helical order, ${ }^{15)}$ studies varying $v_{\mathrm{NNN}}$ are interesting. The local chiral order does not hold the net chirality but contains two types of linear domains (chains) of opposing local chiralities with equal amounts. This suggests that the preference for the twisted arrangement is insufficient to stabilize any chiral phase. It is however noteworthy concerning issues of the chirality and its spatial order that we cannot rule out the possibility that a higher degree of some chiral order is suppressed in the present simulations owing to the finite size effects suffered from the periodic boundary condition imposed.

\subsection{Implication on real liquid crystalline systems}

A computational work performed in this study has revealed the effects of the preference for locally twisted arrangements and phase diagrams with/without the next-nearest-neighbor interaction on lattice models. Here, putting these results in mind, we make comments on real systems where no lattice exists inherently.

Recently, an increasing number of exotic phases has been reported for compounds, which are seemingly rodlike in a broad sense. A gyroid phase is a representative example of such phases. ${ }^{16)}$ The present authors ${ }^{17)}$ have experimentally revealed for a mesogenic series of compounds that, on average, the orientations of neighboring molecules are continuously twisted along bars of two interwoven jungle gyms with opposing handedness, as shown in Fig. 13. Other examples of exotic phases include another cubic phase with chirality 


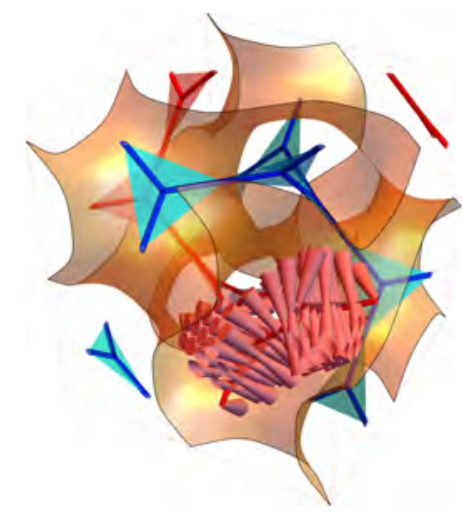

Fig. 13. (Color online) Unit cell of the gyroid phase, based on the X-ray result. ${ }^{17)}$ Two jungle gyms are embedded in two subspaces separated by the gyroid surface, a triply periodic minimal surface. Molecules are arranged along rods of jungle gyms with continuous twists with opposing senses. The "expected" molecular arrangement is drawn between two neighboring junctions in a subspace.

and chiral liquid. ${ }^{7-10,18)}$ Many researchers in the materials development field have intuitively suggested a possible cause of the formation of such phases: An antispindle shape (see Fig. 14) originating from the thermal excitation of conformational disorder of terminal alkyl chain(s) attached to mesogenic molecules causes severe packing frustration. ${ }^{19)}$ In this context, the change in the interaction function expressed by Eq. (2) can intuitively be correlated to that in the molecular shape as in Fig. 14. That is, the antispindle molecular shape is correlated with $r>r_{\mathrm{c}}$.

The effect of the preference for twisted arrangement is, at least in low temperatures, not negligible even if it is weak. It is consequently better to imagine always twisted alignment for close neighbors. Then, we must accept the emergence of an additional degree of freedom, i.e., the sense (handedness) of twist. At this point, we can point out the similarity between the supposed situation and the model of an ensemble of entities in a single-particle potential that is symmetrical with double wells. If the degree of the antispindle shape becomes large, the free choice of the twist sense is prohibited by the next-nearest-neighbor interaction. That is, the interaction works between particles. Such systems consisting of interacting particles are known to undergo a phase transition to a symmetry-broken state on cooling. ${ }^{20,21)}$ Indeed, we have found, in the lattice models, the alternate order to reduce the frustration originating in the local twists. We thus suggest that ensembles of molecules with an antispindle shape with some sufficient degree will exhibit a phase sequence, on cooling, such as isotropic liquid (IL) $\rightarrow$ nematic liquid crystal $\rightarrow$ symmetry-broken phase concerning the twist, even if they have no lattice structure inside.

The simulations indicated that no spatial order concerning the local chirality occurs without the next-nearest-neighbor interaction, whereas a spatially linear segregation of the local chirality may be induced with it. It is thus interesting to remember the gyroid phase (Fig. 13). The gyroid phase is the bicontinuous cubic phase most widely observed ${ }^{16)}$ in soft matter ranging from polymers with a cell constant in micrometer scale ${ }^{22)}$ to thermotropic and lyotropic liquid crystals with that in nanometer scale. ${ }^{23,24)}$ The two subspaces divided by

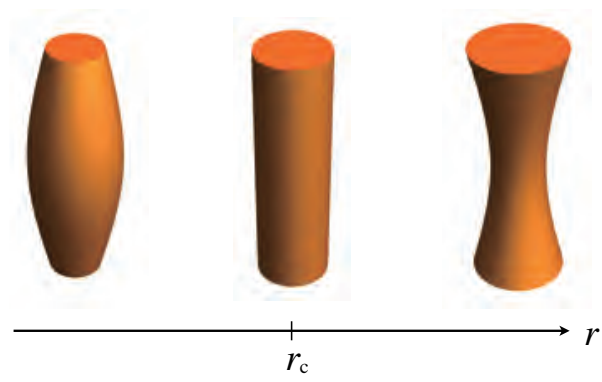

Fig. 14. (Color online) Image of the geometrical change (from spindle to antispindle via cylinder) for a nonpolar molecule with the axial symmetry. $r_{\mathrm{c}}$ $\left[=\frac{9}{79}\right.$ for the potential expressed by Eq. (2)] is a threshold for twist.

the gyroid surface are physically equivalent, but have opposing handedness, as evidenced by the direct observation under electron microscopy. ${ }^{25)}$ The molecular arrangement in the gyroid phase exhibited by a series of thermotropic mesogens has recently been revealed to be continuously twisted throughout the space, as schematically shown in Fig. 13. ${ }^{17)}$ The twist sense of the molecular arrangement on a jungle gym is opposing in two subspaces, accordingly. Because of their ubiquitous presence, some mechanisms have been suggested to rationalize the formation of the gyroid phase. ${ }^{16,26-31)}$ Note that they are mostly independent and do not interfere with one another, leading to a possibility of their cooperation to stabilize real gyroid phases. Thus, if we once accept such mechanisms, it is possible to imagine a virtual lattice structure on jungle gyms. This prompted us to discuss gyroid phases on the basis of the present simulation results. Since the linear segregation of domains with opposing local chirality with equal volumes is an important feature of the gyroid phase, it is plausible that this might come from the antispindle shape of molecules assuming the presence of some next-nearest-neighbor interaction, as naïvely and rather widely assumed. ${ }^{19)}$ Since constituting molecules are seemingly achiral in real systems, it is preferable for molecules to have the same volume for opposing twists. This is guaranteed in the gyroid phase. The equal preference for the two handedness of twisted molecular arrangement is therefore identified as another microscopic origin of the superior stability of the gyroid phase, in addition to the factors previously identified by existing treatments. ${ }^{16,26-31)}$ It is noteworthy that the achiral space group $P 2_{1} / c$, which is the most efficient for dense packing ${ }^{32)}$ and observed most often for usual crystals of achiral molecules, ${ }^{33)}$ shares, with the gyroid case, the local chiral arrangement with equal frequencies for opposing handedness.

According to the simulation results, a phase exhibiting net chirality is not formed although the nanosegregation of local chirality may be induced. This suggests that the chirality of a molecule itself is necessary for such phases. Recently, the chiral "Im3m phase" and optically active isotropic liquid, both made of seemingly and dynamically achiral molecules, were reported. $^{7-10,18)}$ On the basis of the present results, we suggest that the flexibility of molecules (twisting degrees of freedom around single bonds) and a resulting adaptive chirality responding to the environment play an important role in the formation of such chiral phases, as naïvely assumed without reasoning by the authors of previous reports. ${ }^{7-9)}$ 


\section{Conclusions}

The effect of the preference for nonparallel alignments of neighboring headless spins is examined by naïvely extending the Maier-Saupe model on a simple cubic lattice. In addition to the disordered and uniaxial (nematic) phases covered by the Maier-Saupe theory, the appearance of symmetry-broken phases concerning the local twist is suggested if only a nearest neighbor interaction is taken into account. Weak next-nearestneighbor interaction induces other instabilities but with a different spatial order depending on its sign. In particular, a possible segregation of the local chirality is demonstrated.

A discussion on real liquid crystalline systems assuming the mapping of interaction potential on molecular shape leads to the finding that the twisted local arrangement and the equality of the volume of two handedness arising from the achirality of constituting molecules just fit the gyroid phase, which is the most often observed among cubic liquid crystals. Although the geometrical frustration due to the antispindle shape of molecules has intuitively been suggested to be a cause for the formation of cubic liquid crystals, the present results provide a strong support for the idea. Furthermore, this finding also provides a microscopic cause for the abundance of the gyroid phase in nature. It is also suggested that the molecular flexibility enabling the adaptive chirality plays an important role in the formation of phases with net chirality.

\section{Acknowledgement}

This work was supported in part by a Grant-in-Aid for Scientific Research (B) (No. 26288031) from the Japan Society for the Promotion of Science.

\section{Appendix}

The preference for twisted alignment $\left(\theta=\frac{\pi}{2}\right)$ of interacting spins can be treated within the (mean-field) Maier-Saupe theory by assuming $V_{0}<0$ in potential function with the form of Eq. (1). This is briefly described together with the mean field treatment for the case of $r=1$.

The Maier-Saupe theory assumes that the interaction between neighboring spins is proportional to $P_{l}\left(\cos \theta_{i, j}\right)$. The addition theorem for spherical harmonics $Y_{l m}(\theta, \phi)$,

$$
P_{l}\left(\cos \theta_{i, j}\right)=\frac{4 \pi}{2 l+1} \sum_{m=-l}^{l} Y_{l m}\left(\theta_{i}, \phi_{i}\right) Y_{l m}^{*}\left(\theta_{j}, \phi_{j}\right),
$$

enables us to put

$$
P_{l}\left(\cos \theta_{i, j}\right)=P_{l}\left(\cos \theta_{i}\right) P_{l}\left(\cos \theta_{j}\right)
$$

as far as the discussion is limited to axially symmetric systems consisting of (axially symmetric) spins (after averaging over $\phi$ ). Since the interaction is approximated by a bilinear product of functions of a variable assigned to a single spin, we request

$$
V_{\mathrm{MF}}(\theta)=\sigma z_{0} V(\theta)
$$

using an order parameter $\sigma$ with $z_{0}$ being the number of interacting molecules as usual. Now, the intermolecular potential is modified as

$$
\frac{V(\theta)}{V_{0}}=a P_{l}(\cos \theta)
$$

using an adjustable parameter $a$. Since $V_{\mathrm{MF}}(\theta)=z_{0} V(\theta)$ should hold at $T=0, \sigma$ is to converge to unity with $T \rightarrow 0$.
The order parameter $\sigma$ is defined as

$$
\sigma=\operatorname{sgn}\left(V_{0}\right) \frac{\int_{0}^{\pi} a P_{l}(\cos \theta) \exp \left[-\beta V_{\mathrm{MF}}(\theta)\right] \sin \theta d \theta}{\int_{0}^{\pi} \exp \left[-\beta V_{\mathrm{MF}}(\theta)\right] \sin \theta d \theta} .
$$

Note that this definition always yields positive $\sigma$. According to this definition, $\sigma=\operatorname{sgn}\left(V_{0}\right) a P_{l}\left(\cos \theta_{\min }\right)$ at $T=0$ with $\theta_{\min }$ that minimizes $V(\theta)$. Thus, it is necessary to put

$$
a=\operatorname{sgn}\left(V_{0}\right) \frac{1}{P_{l}\left(\cos \theta_{\min }\right)} .
$$

Since $\theta_{\min }$ differs depending on the sign of $V_{0}, a$ should be chosen depending on the sign of $V_{0}$. Setting with $l=2$, $V_{0}>0$, and $a=1$ corresponds to the original Maier-Saupe theory. ${ }^{1)}$

For the antinematic order with the negative nematic order parameter with $l=2\left(\theta_{\min }=\frac{\pi}{2}\right), a=2$ is necessary. The numerical solution of Eq. (A.1) yields a single phase transition around $T \approx 0.80 z_{0} V_{0}$ between the disordered (isotropic) phase and the uniaxial (nematic) phase with a negative nematic order parameter $\left\langle P_{2}(\cos \theta)\right\rangle=-\frac{\sigma}{2}<0$. The transition temperature normalized by $a V_{0}$ is nearly twice as high as that in the case of $V_{0}>0\left(T_{\mathrm{NI}} \approx 0.22 z_{0} V_{0}\right)$. Since the orientational freedom with $\theta=\frac{\pi}{2}$ does not accompany any energetic cost, the heat capacity at $T=0$ is $\frac{1}{2}$, in contrast to 1 in the case of positive $V_{0}$.

Similarly, in the case of $r=1$ [Eq. (2)], $a=\frac{7}{3}$ is necessary. The numerical solution of Eq. (A-1) in this case yields a single phase transition around $T \approx 0.60 z_{0} V_{0}$.

Note that both estimates for $\theta_{\min } \neq 0\left(V_{0}<0\right.$ and $l=2$, and $r=1)$ are much higher than the transition temperatures obtained in the simulation, although the estimate of the transition temperature is fairly good for $r=0\left[T_{\mathrm{NI}}\left(=T_{\mathrm{du}}\right)\right.$ $\approx 0.22 z_{0} V_{0}$ with $\left.z_{0}=6\right]$.

1) W. Maier and A. Saupe, Z. Naturforsch. A 13, 564 (1958).

2) P. A. Lebwohl and Q. Lasher, Phys. Rev. A 6, 426 (1972).

3) H. Meirovitch, Chem. Phys. 21, 251 (1977).

4) P. G. de Genne and J. Prost, The Physics of Liquid Crystals (Clarendon Press, Oxford, 1993) 2nd ed.

5) R. L. Humphries, P. G. James, and G. R. Luckhurst, J. Chem. Soc. Faraday Trans. II, 68, 1031 (1972).

6) Y. R. Lin-Liu, Y. M. Shih, and C.-W. Woo, Phys. Rev. A, 15, 2550 (1977).

7) C. Dressel, F. Liu, M. Prehm, X. Zeng, G. Ungar, and C. Tschierske, Angew. Chem. Int. Ed. 53, 13115 (2014).

8) C. Dressel, T. Reppe, M. Perhm, M. Brautzsch, and C. Tschierske, Nat. Chem. 6, 971 (2014).

9) C. Tschierske and G. Ungar, ChemPhysChem 17, 9 (2016).

10) S. Kutsumizu, S. Miisako, Y. Miwa, M. Kitagawa, Y. Yamamura, and K. Saito, Phys. Chem. Chem. Phys. 18, 17341 (2016).

11) J. -P. Lü and Q.-H. Chen, Commun. Theor. Phys. 57, 166 (2012).

12) G. A. Canova, Y. Levin, and J. J. Arenzon, Phys. Rev. E 94, 032140 (2016).

13) N. Metropolis, A. W. Rosenbluth, M. N. Rosenbluth, A. H. Teller, and E. Teller, J. Chem. Phys. 21, 1087 (1953).

14) C. Dasgupta and B. I. Halperin, Phys. Rev. Lett. 47, 1556 (1981).

15) A. Yoshimori, J. Phys. Soc. Jpn., 14, 807 (1959).

16) S. Hyde, S. Andersson, K. Larsson, Z. Blum, T. Landh, S. Lidin, and B. W. Ninham, The Language of Shape (Elsevier, Amsterdam, 1997).

17) Y. Nakazawa, Y. Yamamura, S. Kutsumizu, and K. Saito, J. Phys. Soc. Jpn. 81, 094601 (2012).

18) K. Saito, Y. Yamamura, Y. Miwa, and S. Kutsumizu, Phys. Chem. Chem. Phys. 18, 3280 (2016). 
19) S. Kutsumizu, H. Mori, M. Fukatami, S. Naito, K. Sakajiri, and K. Saito, Chem. Mater. 20, 3675 (2008).

20) Y. Onodera, Prog. Theor. Phys. 44, 1477 (1970).

21) K. Saito, J. Phys. Soc. Jpn. 67, 3137 (1998).

22) I. W. Hamley, The Physics of Block Copolymers (Oxford University Press, Oxford, 1998).

23) J. W. Goodby, P. J. Collings, T. Kato, C. Tschierske, H. F. Gleeson, and P. Raynes (eds), Handbook of Liquid Crystals Vol. 5 Non-Conventional Liquid Crystals (Wiley, Weinheim, 2014).

24) A. M. F. Neto and S. R. A. Salinas, The Physics of Lyotropic Liquid Crystals (Oxford University Press, London, 2005).

25) H. Jinnai, T. Kajihara, H. Watashiba, Y. Nishikawa, and R. J. Spontak, Phys. Rev. E 64, 010803(R) (2001).
26) L. Leibler, Macromolecules 13, 1602 (1980).

27) T. Ohta and K. Kawasaki, Macromolecules 19, 2621 (1986).

28) M. W. Matsen and F. S. Bates, Macromolecules 29, 1091 (1996).

29) V. E. Podneks and I. W. Hamley, JETP Lett. 64, 617 (1996).

30) L. J. Ellison, D. J. Michel, F. Barmes, and D. J. Cleaver, Phys. Rev. Lett. 97, 237801 (2006).

31) K. Saito, Y. Yamamura, and S. Kutsumizu, J. Phys. Soc. Jpn. 77, 093601 (2008).

32) A. I. Kitaigorodsky, Molecular Crystals and Molecules (Academic Press, New York, 1973).

33) CSD Space Group Statistics 2015, the statistics of the Cambridge Structural Database as of November 6, 2015, available at http://ccdc.cam.ac.uk (The Cambridge Crystallographic Data Centre). 